fashion. The sheer dimensions of this compilation and the inaccuracies pointed out should, on the other hand, serve to encourage China specialists from various disciplines to combine their efforts in future similar projects. No single compiler is likely to command the expertise necessary for lucid and well-thought-out translations and explanations of all the terminology assembled between the convers of this, on the whole, remarkable dictionary.

Wolfgang Keßler

\title{
Jeanette Greenfield
}

China and the Law of the Sea, Air, and Environment

Sijthoff \& Noordhoff, Alphen aan den Rijn u. Germantown, Maryland - USA, 1979, US $\$ 32.50$

Seit der Aufnahme der Volksrepublik China (VRC) in die Vereinten Nationen im Oktober 1971 ist die Haltung Chinas hinsichtlich ínternational-rechtlicher Fragen als wichtiger Faktor der Fortbildung des Völkerrechts für die übrigen Mitglieder der Staatengemeinschaft von wachsender Bedeutung. Ein u. a. auf den Gebieten des See-, Luft- und Umweltrechts diesbezüglich bestehendes Informationsdefizit will das vorliegende Buch von Jeanette Greenfield abbauen.

In ihrer Untersuchung wertet die Autorin neben der nur spärlich vorhandenen Literatur Stellungnahmen der VRC, insbesondere in Ausschüssen und Organen der Vereinten Nationen, völkerrechtliche Verträge, an denen sie beteiligt ist, einseitige Deklarationen sowie Veröffentlichungen in der chinesischen Presse als amtlichen Publikationsorganen aus. Die schwer auffindbaren Originärquellen, z. T. auch nationale chinesische Rechtsnormen, sind teilweise in einem umfangreichen Anhang (93 Seiten) abgedruckt, u. a. die Declaration on China's Territorial Sea v. 4. 9. 1958, Communist China Regulations on Rivers and Ports, PRC Regulations for Waterway and Cargo Transportation, ferner verschiedene von der chinesischen Delegation im "Committee on the peaceful use of the sea-bed and the ocean floor beyond the limitis of national jurisdiction" eingebrachte Arbeitspapiere.

Der Leser, der eine klare Aussage erwartet, inwieweit die chinesische Rechtsauffassung mit universellem Völkerrecht übereinstimmt oder davon abweicht, wird jedoch enttäuscht. Es gelingt der Autorin nicht, die zahlreichen Einzeläußerungen, die nach ihrem eigenen Resumé z. T. recht widersprüchlich sind, in übersichtlicher Form zu ordnen und darzustellen. Ein Gliederungssystem innerhalb der einzelnen Kapitel sucht man vergeblich. Die durch die Voranstellung eines kurzen Abrisses chinesischer Rechtsgeschichte, verbunden mit dem Hinweis auf die Verknüpfung von Recht und Philosophie, geweckte Erwartung, in den folgenden Artikeln eine Bezugnahme, bzw. Erklärungsversuche oder Erläuterungen zu finden, wie sich die völkerrechtlichen Grundtendenzen der VRC aus 
der älteren und jüngeren Geschichte erklären lassen, erfüllt sich nicht. Schlußfolgerungen, wie etwa, daß es Verbindungslinien geben könnte zwischen dem ausgeprägten Souveränitatsdenken des kommunistischen China und dem noch Anfang des Jahrhunderts virulenten $\mathrm{Ha}$ gegen Fremdherrschaft, bleiben dem Leser selbst überlassen.

Das Buch ist gegliedert in drei Hauptabschnitte: See, Luft und Umwelt. Den meisten Raum nehmen erwartungsgemäß seerechtliche Fragen ein. Als dominierender Grundzug wird hier die mangelnde Bereitschaft hervorgehoben, Hoheitsrechte in irgendeiner Weise beschneiden zu lassen oder freiwillig zu beschränken. Ein Zeichen dieser Bindungsunwilligkeit ist auch die Tatsache, daß die VRC zwar an diversen Konferenzen zur Kodifizierung des Völkerrechts in den hier zur Debatte stehenden Gebieten teilgenommen hat, jedoch eine Unterzeichnung letztlich immer ablehnte, sei es unter Hinweis auf ein von ihr nicht gebilligtes Verfahren bei der Ausarbeitung des Textes, sei es wegen Abweichungen der eigenen Auffassungen von den vereinbarten Regelungen, seien sie auch noch so geringer Art.

Das besondere Engagement Chinas gerade auf dem Gebiet des Seevölkerrechts, führt die Autorin auf drei Hauptursachen zurück, nämlich Erdölvorkommen in für China interessanten Gebieten, einer Identifizierung der VRC mit Interessen der Entwicklungsländer und die wachsende Rivalität zur UdSSR. In allen Kapiteln des ersten Teils (Eigengewässer, Küstengewässer, Anschluß- oder Wirtschaftszone, Hohe See, Festlandsockel) wird das Reklamieren extensiver Hoheitsgewalt exemplifiziert, als wesentlichste Abweichung chinesischer Praxis von internationalen Normen hervorgestrichen. So werden die nach geltendem Völkerrecht nicht begründbaren Ansprüche der VRC auf bestimmte Gewässerteile (Pohay-Bay, Chiung Chou-Meerenge etc.) dargestellt. Als weiteres Beispiel dient der chinesische Anspruch auf Achtung von über das allgemeine Völkerrecht hinausgehenden Gebietshoheitsrechten. Die VRC fordert z. B. uneingeschränkt die Einholung einer Genehmigung zur Kabelverlegung im als Festlandsockel vom Küstenstaat beanspruchten Gebiet. Auch das Recht auf innocent passage wird durch Errichtung von "Schutzzonen" in Küstengewässern beschnitten.

Auch an der Festlandsockeldoktrin wurde von chinesischer Seite heftige Kritik geübt. Als weitere Abweichung von der völkerrechtlich vorherrschenden Praxis wird angeführt, daß die VRC sowohl die Grenzfestlegung der zur Wirtschaftszone ausgedehnten Küstengewässer und Anschlußzone (wobei 200 Meilen als zulässig, aber nicht als äußerste Grenze betrachtet werden) als auch die des Festlandsockels als allein Sache des Küstenstaates begreift. Vom chinesischen Standpunkt abweichende Normen werden als von den starken Seemächten erzeugtes, dritte Parteien nicht bindendes Recht betrachtet. Auf die Ablehnung einer Bindung neu entstandener oder unabhängig gewordener Staaten durch früher zustandegekommene Verträge und Gewohnheitsrecht weist die Autorin an verschiedenen Stellen hin. Man vermißt hierbei eine Erwähnung der sog. "ungleichen Verträge", durch die China seit dem Frieden von Nanking 1842 jahrzehntelang gedemütigt wurde.

Die Gesamtbeurteilung der Autorin, im wesentlichen weiche die Haltung der VRC zu seevölkerrechtlichen Fragen nicht nennenswert von den Prinzipien geltender seevölker- 
rechtlicher Normen ab, erstaunt angesichts der zahlreichen dargestellten entgegenstehenden Beispiele.

Im das Luftrecht behandelnden zweiten Teil, der mit 10 Seiten recht knapp gehalten ist, wird vorwiegend dargestellt, welche bilateralen Bindungen die VRC eingegangen ist. Grundsätzlich hält die VRC, ihrem Sicherheitsbedürfnis entsprechend, strikt an ihrer ausschließlichen, auch der Höhe nach als unbegrenzt und unbegrenzbar angesehenen Territorialhoheit über dem Luftraum fest. Für vertraglich eingeräumte Úberflug- und Zwischenlandungsrechte gelten strenge Begrenzungen und Sicherheitsbestimmungen. Multilateralen Verträgen, aus denen sich auch Verpflichtungen, insbesondere auf haftungsrechtlichem Gebiet ergeben hätten, ist die VRC nicht beigetreten.

Vergleichsweise ebenfalls zu kurz kommt der unter der vielversprechenden Úberschrift "Environment" ganze 20 Seiten umfassende dritte Teil, der die Frage der Meeresverschmutzung nur anreißt und die atmosphärische Verschmutzung nur unter dem Gesichtspunkt der Gefährdung durch Atomwaffen behandelt. Angesichts dieser Selbstbeschränkung erstaunt die Aufnahme eines Teils "Bevölkerung" als Umweltverschmutzungsproblem. Ein Zusammenhang zwischen Bevölkerungswachstum und Umweltschäden wird von der VRC negiert, weil die Eröffnung und Nutzung natürlicher Ressourcen durch die Menschheit sich ständig ausweite. Daß die von der Autorin zunächst angeführte Begründung für diese Haltung (die Ausbeutung natürlicher Quellen sei unerschöpflich), auf einer fehlerhaften und teils unrichtig übernommenen Ubersetzung der hierfür zitierten Quelle beruht, wird erst im Schlußwort (S. 234/35) erwähnt. Hiermit verbindet die Autorin gleichzeitig den für jeden an chinesischer Völkerrechtsauffassung Interessierten wichtigen Hinweis, daß eine der Hauptschwierigkeiten im Verständnis der Haltung der VRC zum Völkerrecht die Gefahr der Mißinterpretation bei der Úbersetzung chinesischsprachiger Äußerungen in westliche Sprachen darstellt.

Karin Kammann

\section{Oskar Weggel}

\section{Chinesische Rechtsgeschichte}

E. J. Brill, Leiden, Köln, 1980, XI, 298 S., Gld. 128.—

Das Werk ist als Sechster Band der Vierten Abteilung ("China") des von dem Hamburger Orientalisten Berthold Spuler herausgegebenen Handbuchs der Orientalistik erschienen. Der Autor, der zu zahlreichen Fragen insbesondere modernen chinesischen Rechts publiziert hat, schildert die Entwicklungsgeschichte des klassischen Rechts von der Zhou-Zeit bis 1911, wobei er den wichtigsten Dynastien jeweils ein Kapitel widmet. Ausgehend vom Recht der Qing-Dynastie (1644-1911) berichtet er sodann vor allem über allgemeines Strafrecht, Verwaltung, Zivilrecht, strafrechtliche Einzeltatbestände. Am Ende dieses Abschnitts faßt Weggel Charakteristika des chinesischen Rechts zusammen, wobei er dessen Beharrungskraft besonders akzentuiert. Knapp 40 Seiten sind schließ- 